\title{
Point defect balance in epitaxial GaSb
}

\author{
N. Segercrantz, ${ }^{1, a)}$ J. Slotte,${ }^{1}$ I. Makkonen, ${ }^{1}$ J. Kujala, ${ }^{1}$ F. Tuomisto, ${ }^{1}$ Y. Song, ${ }^{2,3}$ \\ and S. Wang ${ }^{2,3}$ \\ ${ }^{1}$ Department of Applied Physics, Aalto University, P.O. Box 14100, FIN-00076 Aalto Espoo, Finland \\ ${ }^{2}$ Department of Microtechnology and Nanoscience, Chalmers University of Technology, 41296 Göteborg, \\ Sweden \\ ${ }^{3}$ State Key Laboratory of Functional Materials for Informatics Shanghai Institute of Microsystem \\ and Information Technology, Chinese Academy of Sciences 865 Changning Road, Shanghai 200050, China
}

(Received 4 July 2014; accepted 21 August 2014; published online 29 August 2014; publisher error corrected 9 September 2014)

\begin{abstract}
Positron annihilation spectroscopy in both conventional and coincidence Doppler broadening mode is used for studying the effect of growth conditions on the point defect balance in $\mathrm{GaSb}: \mathrm{Bi}$ epitaxial layers grown by molecular beam epitaxy. Positron annihilation characteristics in GaSb are also calculated using density functional theory and compared to experimental results. We conclude that while the main positron trapping defect in bulk samples is the Ga antisite, the Ga vacancy is the most prominent trap in the samples grown by molecular beam epitaxy. The results suggest that the $p$-type conductivity is caused by different defects in GaSb grown with different methods. (C) 2014 AIP Publishing LLC. [http://dx.doi.org/10.1063/1.4894473]
\end{abstract}

GaSb has a narrow direct band gap, high electron mobility, and can be lattice matched to its related ternary and quaternary compounds making it a suitable candidate for optoelectronic devices as well as for high speed electronics. ${ }^{1,2}$ Regardless of growth method, undoped GaSb is unintentionally $p$-type. ${ }^{1}$ Many efforts have been made to reduce the $p$-type conductivity in GaSb. By growing GaSb from Sb-rich melts, the residual hole concentration was shown to decrease from the usually reported value of $10^{17}$ to $10^{16} \mathrm{~cm}^{-3}$. 3 Anayama et $a l .{ }^{4}$ reported a lowered $p$-type conductivity as a result of a decreased growth temperature for liquid-phase-epitaxy-grown $\mathrm{GaSb}$ from Sb-rich solutions. Similar results were achieved by $\mathrm{Li}$ et al..$^{5}$ Reducing the growth temperature or the $\mathrm{Sb} / \mathrm{Ga}$ flux for molecular beam epitaxy (MBE) grown GaSb epitaxial layers led to a decrease in the acceptor concentration. It was suggested that an increased growth temperature increases the evaporation of $\mathrm{Sb}$ atoms, which in turn leads to an increase in $\mathrm{Sb}$ lattice sites available for $\mathrm{Ga}$ atoms.

Not many reports exist on the growth ${ }^{6,7}$ and properties of $\mathrm{GaSb}_{1-\mathrm{x}} \mathrm{Bi}_{\mathrm{x}}$, one of the newer members of the dilute bismide family to be studied. A few studies ${ }^{8,9}$ reporting a decreased residual hole concentration in $\mathrm{GaSb}$ due to incorporation of Bi have been published. In addition, some reports state a modified defect enviroment ${ }^{10}$ and an improved crystal quality $^{11}$ of MBE-grown GaAs when dilute amounts $(<1 \%)$ of $\mathrm{Bi}$ is added. The usage of $\mathrm{Bi}$ as a surfactant during growth has also been somewhat modestly studied. Bi has been reported to improve the structural and optical quality of InGaAs/GaAsBi structures. ${ }^{12}$ Also, a reduced defect incorporation in MBE-grown dilute GaNAs alloys was achieved by applying a Bi flux during growth. ${ }^{13}$

During the last decades, positron annihilation spectroscopy (PAS) has been employed for studying the defects in GaSb. ${ }^{14-21} \mathrm{PAS}$ is a versatile tool for studying point defects in semiconductors. ${ }^{22}$ Its power is due to the selective sensitivity

a)natalie.segercrantz@aalto.fi to vacancy defects and the insensitivity to conductivity and band gap width. It is particularly suitable for studying narrow band gap semiconductors such as GaSb. From positron lifetime spectroscopy (PALS) on electron-irradiated liquid encapsulated Czochralski-grown GaSb, Ling et al. ${ }^{14}$ and later Ma et al., ${ }^{15}$ characterized two different gallium vacancy $\left(V_{\mathrm{Ga}}\right)$-related defects with different microstructures. In the same reports, an acceptor believed to be responsible for the $p$-type conduction in $\mathrm{GaSb}$ was identified from photoluminescence and temperature dependant Hall measurements. The defect was not related to the Ga vacancy. As a possible explanation for the $p$-type behavior in GaSb, gallium antisites $\left(\mathrm{Ga}_{\mathrm{Sb}}\right)$ were suggested. Calculations performed by Virkkala et al. ${ }^{23}$ using the density functional theory (DFT) framework showed that the $\mathrm{Ga}_{\mathrm{Sb}}$ antisite defect has one of the lowest formation energies in GaSb. Kujala et al. ${ }^{21}$ performed temperature dependent PALS measurements as well as temperature dependent coincident Doppler broadening measurements on undoped GaSb of $p$-type and Te-doped, $n$-type GaSb. The results showed that the main defect responsible for the acceptor type behavior in bulk GaSb is $\mathrm{Ga}_{\mathrm{Sb}}$, and that these antisites compete with the Ga vacancies in trapping positrons well above room temperature.

In this letter, we use PAS in Doppler broadening mode to study the balance between point defects in MBE-grown $\mathrm{GaSb}_{1-\mathrm{x}} \mathrm{Bi}_{\mathrm{x}}$ epitaxial layers. Also, positron annihilation characteristics in GaSb are calculated using DFT and compared to experimental results. It can be concluded that whereas the $\mathrm{Ga}$ antisite is the main trapping defect and the source of the residual hole concentration in Czochralskigrown bulk $\mathrm{GaSb}$, the Ga vacancy concentration is higher in MBE-grown GaSb and can therefore play a more significant role in the acceptor concentration.

The epitaxial layers were grown on undoped (100) $\mathrm{GaSb}$ substrates in a Riber Compact21 MBE system with a cluster tool, equipped with a dual filament effusion cell for $\mathrm{Ga}$, a single filament effusion cell for $\mathrm{Bi}$ and a valved cracker for $\mathrm{Sb}$. 
A very thin $\mathrm{GaSb}$ buffer layer $(5 \mathrm{~nm})$ was first grown at $510^{\circ} \mathrm{C}$ (measured by a thermocouple) after deoxidation of the GaSb substrate at $580^{\circ} \mathrm{C}$. Reflection high-energy electron diffraction (RHEED) showed a sharp $1 \times 3$ pattern at this stage indicating a smooth growth front. After this, a $200 \mathrm{~nm}$ $\mathrm{GaSb}_{1-\mathrm{x}} \mathrm{Bi}_{\mathrm{x}}$ layer was grown with a growth rate of $0.1 \mu \mathrm{m} / \mathrm{h}$. The beam equivalent pressure (BEP) of $\mathrm{Sb}$ was kept constant at $8.8 \times 10^{-8}$ Torr for all samples. The Bi BEP varied with values close to the $\mathrm{Bi}$ vapor pressure at particular growth temperatures, i.e., $1 \times 10^{-8}, 4 \times 10^{-8}, 7 \times 10^{-8}, 1 \times 10^{-7}$, and $2 \times 10^{-7}$ Torr at $330,360,370,380$, and $390^{\circ} \mathrm{C}$, respectively. More details about the growth can be found in Ref. 6. The Bi concentration in the samples was measured using Rutherford backscattering spectrometry (RBS). The Bi concentration varied between $0 \%$ and $0.1 \%$ in the epitaxial layers.

In order to study the point defect balance in GaSb, conventional as well as coincidence Doppler broadening PAS measurements were carried out. For the conventional Doppler broadening measurements, a variable energy $(0.5-25 \mathrm{keV})$ slow positron beam was used. The setup was equipped with a $\mathrm{HP}$ Ge detector that had an energy resolution of $1.15 \mathrm{keV}$ at $511 \mathrm{keV}$. The window of the $S$ parameter for the measurements reported in this letter was set to $|p|<0.4$ a.u., for the $W$ parameter the window was 1.6 a.u. $<|p|<4.0$ a.u. The $S$ parameter is defined as the ratio of annihilation events in the central, low momentum region of the $511 \mathrm{keV}$ peak whereas the $W$ parameter describes the ratio of events in the high momentum region.

The coincidence Doppler broadening measurements were carried out in a variable energy slow positron beam equipped with two HPGe detectors. In coincidence Doppler, both annihilation photons are detected and the signal is only stored if the photons reach the detectors simultaneously. This enables more detailed studies of the chemical surrounding due to a lower peak-to-background ratio. The resolution of the HPGe-detector was $1.24 \mathrm{keV}$ at $511 \mathrm{keV}$. For statistical reliability, $10^{6}$ and $10^{7}$ annihilation events were collected for each measurement point for the conventional and coincidence measurements, respectively. More details on the experimental technique can be found in Ref. 22.

Doppler broadening spectra of positron annihilation in a defect-free GaSb lattice as well as at different kinds of defect structures were calculated. The technical details of the formalism used can be found in Ref. 24. To summarize, the positron annihilation parameters were modeled using electronic structure calculations; the valence electron densities were obtained self-consistently via the local-density approximation (LDA), employing the projector augmented-wave (PAW) method ${ }^{25}$ and the plane-wave code VASP. ${ }^{26,27}$ The positron states and annihilation characteristics were determined using the $\mathrm{LDA}^{28}$ and the state-dependent scheme ${ }^{27}$ for the momentum densities of annihilating electron-positron pairs. All electronic structure calculations were performed using a 216-atom GaSb zincblende supercell. The defect structures were relaxed taking into account the forces exerted on the ions by the localized positron. The Doppler spectra were computed using the all-electron valence wave functions of the PAW method ${ }^{29}$ and atomic orbitals for the core electrons. The calculated spectra were then convoluted with the resolution of the Doppler measurements.
Figure 1 illustrates the Doppler broadening parameter $S$ as a function of positron implantation energy for the $\mathrm{GaSb}_{1-\mathrm{x}} \mathrm{Bi}_{\mathrm{x}}$ epitaxial layers. The mean positron implantation depth is also indicated in the figure. At a positron implantation energy in the vicinity of $5 \mathrm{keV}$, most of the detected intensity is coming from positrons annihilating in the $\mathrm{GaSb}_{1-\mathrm{x}} \mathrm{Bi}_{\mathrm{x}}$ epitaxial layer. At these energies, a clear peak in the $S$ parameter curve can be seen in Fig. 1. The highest $S$ parameter corresponds to sample \#1 that has been grown without $\mathrm{Bi}$. Adding Bi during growth seems to reduce the $S$ parameter of the epitaxial layers. For a positron localized at a vacancy, the overlap with core electrons is reduced, resulting in a narrower annihilation peak and a higher $S$ parameter compared to the defect-free lattice. Therefore, the decrease in the $S$ parameter indicates a reduction in the fraction of positrons annihilating at vacancy defects. This could either be due to a reduced vacancy concentration or an increase in positrons trapping into $\mathrm{Ga}$ antisites. As mentioned earlier, the main defect trapping positrons in the Czochralski-grown bulk samples are $\mathrm{Ga}$ antisites, the concentration of $\mathrm{Ga}_{\mathrm{Sb}}$ being roughly one magnitude higher than the $\mathrm{V}_{\mathrm{Ga}}$ concentration.

From the $W(S)$ plot shown in Fig. 2, information on the different positron annihilation states in the samples can be obtained. In this figure, the characteristic $(S, W)$ points for the three annihilation states are illustrated. Hence, the positrons implanted in the sample annihilate in three states: the surface, the epitaxial layer, and the substrate. The annihilation state of the epitaxial layer constitutes a turning point when moving along the measured results from the surface state to the substrate. A sharp turning point in the $S-W$ plane indicates a shorter positron diffusion length and stronger positron trapping. ${ }^{30}$ For the sample denoted \#4, e.g., the turning point is very blunt, whereas for the sample \#1 that has been grown without $\mathrm{Bi}$ the turning point is quite sharp. This gives further evidence that the fraction of positrons trapping into vacancies in the samples grown under a $\mathrm{Bi}$ flux decreases compared to the epitaxial layer grown without $\mathrm{Bi}$.

In Fig. 3, ratio curves from coincidence Doppler broadening measurements are shown. For probing the epitaxial

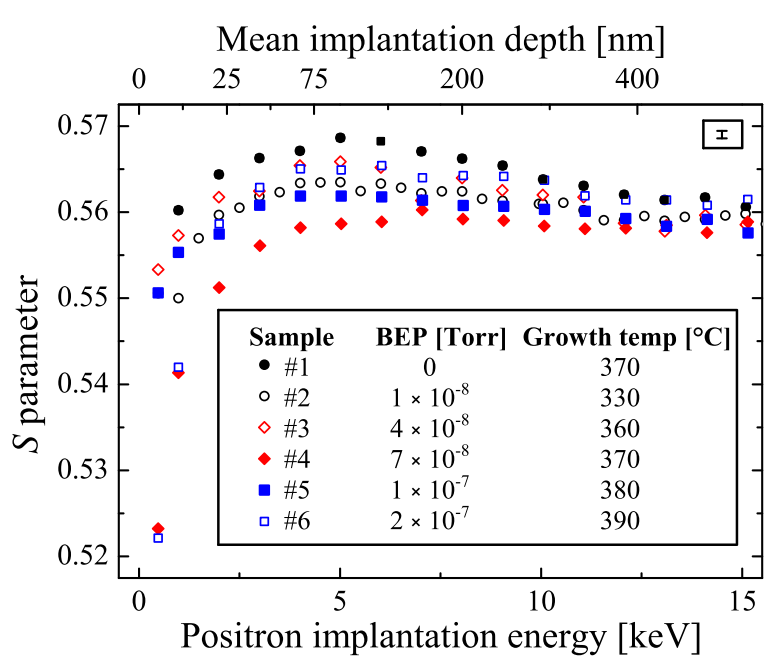

FIG. 1. The $S$ parameters as a function of positron implantation energy for the MBE-grown epitaxial layers. The mean positron implantation depth is also indicated. Typical error bar of the data points is shown in the top right corner. 


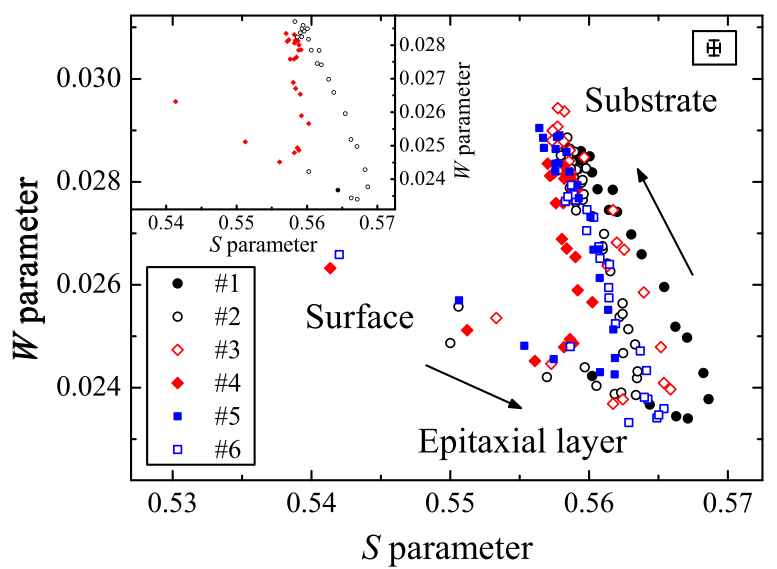

FIG. 2. $W(S)$ plot for the MBE-grown epitaxial layers. The characteristic $(S, W)$ points are indicated. The arrows indicate increasing positron implantation energy. The inset represents the $W(S)$ plot for samples \#1 and \#4. Typical error bar of the data points is shown in the top right corner.

layer, a positron implantation energy of $5 \mathrm{keV}$ was chosen. The data are scaled to undoped, $p$-type GaSb measured at room temperature in Ref. 21. GaSb of $p$-type was chosen as the reference because of its lower positron lifetime compared to that of the $n$-type GaSb. The Ga vacancy concentration for $p$-type $\mathrm{GaSb}$ was estimated to be $3 \times 10^{16} \mathrm{~cm}^{-3}$, the $\mathrm{Ga}$ antisite concentration $2 \times 10^{17} \mathrm{~cm}^{-3}$. Fig. 4 illustrates the computed momentum distributions of the $\mathrm{Ga}$ and the $\mathrm{Sb}$ vacancies scaled to that of the defect-free GaSb lattice. The experimental result obtained for sample \#1 is also shown.

As can be seen from Fig. 3, the ratio curves of the MBE-grown epitaxial layers look completely different compared to the $p$-type GaSb used as a reference. At low momenta, the intensity is higher corresponding to a higher $S$ parameter and at momenta near 1.2 a.u. an intensity peak for the ratio curves can be seen. Compared to the $p$-type $\mathrm{GaSb}$ bulk used as a reference, the higher $S$ parameter indicates that the trapping into vacancies is more dominant in the epitaxial layers.

From Fig. 4, it can be concluded that the calculated momentum distribution of the Ga vacancy scaled to the defectfree lattice agrees best with the measured results. This is in

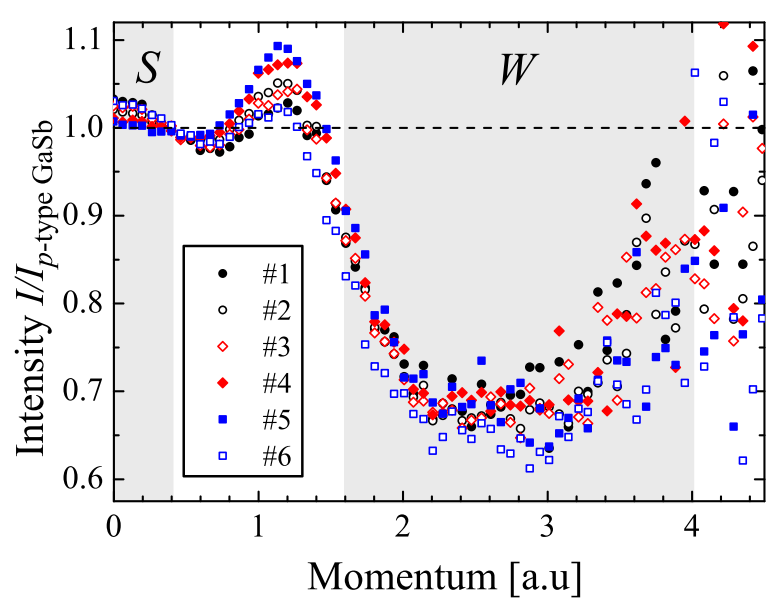

FIG. 3. Ratio curves of the intensity from coincidence Doppler measurements. The data are scaled to $p$-type bulk GaSb indicated by the dashed line. ${ }^{21}$ The windows of the line shape parameters are sketched.

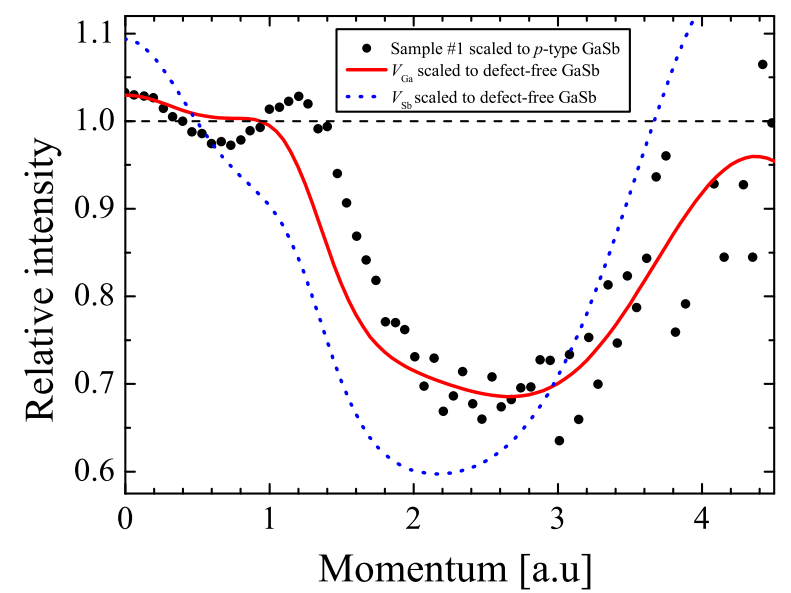

FIG. 4. Calculated momentum distribution ratio curves for the $V_{\mathrm{Ga}}$ and $V_{\mathrm{Sb}}$ defects compared to a measured result. The dashed line indicates the reference.

line with earlier studied, e.g., diffusion experiments showing that the $\mathrm{V}_{\mathrm{Sb}}$ defect in $\mathrm{GaSb}$ is unstable. ${ }^{31}$ Also, theoretical calculations indicate that the $\mathrm{Sb}$ vacancy is positive and thereby undetectable with PAS. ${ }^{23}$ The DFT calculations show that as the positron is localized at the Ga vacancy, less of the signal comes from annihilations with $\mathrm{Ga} 3 d$ electrons and more from the $\mathrm{Sb} 4 d$ electrons causing a higher $S$ parameter and the shoulder at 1.2 a.u. seen in Fig. 4. The dip in the spectra at higher momenta $(p>2$ a.u.) is caused by a decrease in annihilations with Ga $3 d$ electrons. The discrepancy between the calculated Ga vacancy ratio curve and measured results at momenta in the vicinity of 1.2 a.u. is due to the different references used. The calculated results are scaled to a defect-free lattice, whereas the experimental results are scaled to $p$-type $\mathrm{GaSb}$ with $\mathrm{Ga}_{\mathrm{Sb}}$ dominating the positron annihilation.

As a $\mathrm{Bi}$ flux is added and the growth temperature increases, the $S$ parameter decreases and the peak at 1.2 a.u. increases. The decrease in the $S$ parameter is due to the decrease in positrons trapping into Ga vacancy as concluded earlier. The increase in the intensity of the peak is partly due to the decreased trapping into Ga vacancies and partly due to the large concentration of $\mathrm{Ga}$ antisites acting as positron traps in the reference sample. From these results, it can be concluded that as the Bi flux and the growth temperature are increased and the $S$ parameter decreases, the Ga vacancy concentration indeed decreases. If the antisite concentration would increase and the trapping to these defects would become more dominant, the ratio curve should coincide more with the $p$-type bulk used as reference.

In conclusion, the PAS results presented in this letter show that the growth conditions have a clear impact on the ratio of the Ga vacancy to the Ga antisite concentration in GaSb. Compared to the Czochralski-grown bulk GaSb samples where the main positron trapping defect is the Ga antisite, the ratio of the Ga vacancy concentration to the $\mathrm{Ga}$ antisite concentration seems to be higher in the MBE-grown samples. When a Bi flux is added and the growth temperature increases, the vacancy concentration of the epitaxial layers decreases. This in line with the earlier studies showing a decreased density of $\mathrm{Ga} /$ As-related defects for dilute 
GaAsBi layers ${ }^{10}$ and a decreased defect incorporation as a result of Bi behaving as a surfactant during growth. ${ }^{12,13}$ Our results indicate that the defect responsible for $p$-type conductivity in GaSb can depend on the growth method. In Czochralski-grown bulk GaSb, the Ga antisite is the main trapping defect and the source of the residual hole concentration. In epitaxial layers, the Ga vacancy may play a more significant role in the acceptor concentration.

We gratefully acknowledge Andres Hallén and Daniel Primetzhofer for performing the RBS measurements on the samples. We would also like to acknowledge Swedish Foundation for Strategic Research (SSF), the National Basic Research Program of China (Grant No. 2014CB643902), and the Key Program of Natural Science Foundation of China (Grant No. 61334004) for financial support. The calculations presented above were performed using computer resources within the Aalto University School of Science "Science-IT" project.

${ }^{1}$ P. S. Dutta, H. L. Bhat, and V. Kumar, J. Appl. Phys. 81, 5821-5870 (1997).

${ }^{2}$ A. G. Milnes and A. Y. Polyakov, Solid State Electron. 36, 803-818 (1993).

${ }^{3}$ F. J. Reid, R. D. Baxter, and S. E. Miller, J. Electrochem. Soc. 113, 713-716 (1966).

${ }^{4}$ C. Anayama, T. Tanahashi, H. Kuwatsuka, S. Nishiyama, S. Isozumi, and K. Nakajima, Appl. Phys. Lett. 56, 239-240 (1990).

${ }^{5}$ Y. Li, Y. Zhang, Y. Zhang, B. Wang, Z. Zhu, and Y. Zeng, Appl. Surf. Sci. 258, 6571-6575 (2012).

${ }^{6}$ Y. Song, S. Wang, I. Saha Roy, P. Shi, and A. Hallen, J. Vac. Sci. Technol. B 30, 02B114 (2012).

${ }^{7}$ M. K. Rajpalke, W. M. Linhart, M. Birkett, K. M. Yu, D. O. Scanlon, J. Buckeridge, T. S. Jones, M. J. Ashwin, and T. D. Veal, Appl. Phys. Lett. 103, 142106 (2013).

${ }^{8} \mathrm{M}$. Kucera and J. Novak, in Proceedings of The Fourth International Conference on Advanced Semiconductor Devices and Microsystems (IEEE, 2002), pp. 149-152.

${ }^{9}$ A. N. Danilewsky, S. Lauer, J. Meinhardt, K. W. Benz, B. Kaufmann, R. Hofmann, and A. Dornen, J. Electron. Mater. 25, 1082-1087 (1996).
${ }^{10}$ A. R. Mohmad, F. Bastiman, C. J. Hunter, J. S. Ng, S. J. Sweeney, and J. P. R. David, Appl. Phys. Lett. 99, 042107 (2011).

${ }^{11}$ P. M. Mooney, K. P. Watkins, Z. Jiang, A. F. Basile, R. B. Lewis, V. Bahrami-Yekta, M. Masnadi-Shirazi, D. A. Beaton, and T. Tiedje, J. Appl. Phys. 113, 133708 (2013).

${ }^{12}$ M. R. Pillai, S.-S. Kim, S. T. Ho, and S. A. Barnett, J. Vac. Sci. Technol. B Microelectron. Nanometer Struct. Process., Meas., Phenom. 18, 1232-1236 (2000).

${ }^{13}$ S. Tixier, M. Adamcyk, E. C. Young, J. H. Schmid, and T. Tiedje, J. Cryst. Growth 251, 449-454 (2003).

${ }^{14}$ C. C. Ling, M. K. Lui, S. K. Ma, X. D. Chen, S. Fung, and C. D. Beling, Appl. Phys. Lett. 85, 384-386 (2004).

${ }^{15}$ S. K. Ma, M. K. Lui, C. C. Ling, S. Fung, C. D. Beling, K. F. Li, K. W. Cheah, M. Gong, H. S. Hang, and H. M. Weng, J. Phys.: Condens. Matter 16, 6205 (2004).

${ }^{16}$ S. Dannefaer, W. Puff, and D. Kerr, Phys. Rev. B 55, 2182 (1997).

${ }^{17}$ J. Mahony, G. Tessaro, P. Mascher, H. Siethoff, and H.-G. Brion, Mater. Sci. Forum 196, 1449-1454 (1995).

${ }^{18}$ J. Kujala, J. Slotte, and F. Tuomisto, J. Phys.: Conf. Ser. 443, 012042 (2013).

${ }^{19}$ C. C. Ling, W. K. Mui, C. H. Lam, C. D. Beling, S. Fung, M. K. Lui, K. W. Cheah, K. F. Li, Y. W. Zhao, and M. Gong, Appl. Phys. Lett. 80, 3934-3936 (2002).

${ }^{20}$ C. C. Ling, S. Fung, C. D. Beling, and W. Huimin, Phys. Rev. B 64, 075201 (2001).

${ }^{21}$ J. Kujala, N. Segercrantz, F. Tuomisto, and J. Slotte, "Native point defects in GaSb" (unpublished).

${ }^{22}$ F. Tuomisto and I. Makkonen, Rev. Mod. Phys. 85, 1583 (2013).

${ }^{23}$ V. Virkkala, V. Havu, F. Tuomisto, and M. J. Puska, Phys. Rev. B 86, 144101 (2012).

${ }^{24}$ I. Makkonen, M. Hakala, and M. J. Puska, Phys. Rev. B 73, 035103 (2006).

${ }^{25}$ P. E. Blöchl, Phys. Rev. B 50, 17953-17979 (1994).

${ }^{26}$ G. Kresse and J. Furthmüller, Phys. Rev. B 54, 11169-11186 (1996).

${ }^{27}$ M. Alatalo, B. Barbiellini, M. Hakala, H. Kauppinen, T. Korhonen, M. J. Puska, K. Saarinen, P. Hautojärvi, and R. M. Nieminen, Phys. Rev. B 54, 2397-2409 (1996).

${ }^{28}$ E. Boroński and R. M. Nieminen, Phys. Rev. B 34, 3820 (1986).

${ }^{29}$ I. Makkonen, M. Hakala, and M. J. Puska, J. Phys. Chem. Solids 66, 1128-1135 (2005).

${ }^{30}$ S.-L. Sihto, J. Slotte, J. Lento, K. Saarinen, E. V. Monakhov, A. Y. Kuznetsov, and B. G. Svensson, Phys. Rev. B 68, 115307 (2003).

${ }^{31}$ H. Bracht, S. Nicols, W. Walukiewicz, J. P. Silveira, F. Briones, and E. E. Haller, Nature 408, 69-72 (2000). 